Supplementary information for:

\title{
Probing weakly hybridized magnetic molecules by single-atom magnetometry
}

Emil Sierda ${ }^{1,2,}$, Micha Elsebach ${ }^{1}$, Roland Wiesendanger ${ }^{1}$ and Maciej Bazarnik ${ }^{1,2}$

${ }^{1}$ Dept. of Physics, University of Hamburg, Jungiusstrasse 11, D-20355 Hamburg, Germany

${ }^{2}$ Institute of Physics, Poznan University of Technology, Piotrowo 3, 60-965 Poznan, Poland

*esierda@physnet.uni-hamburg.de

\section{Adsorption of CoSal molecules on graphene}

A close-packed highly ordered CoSal molecular assembly on pristine GR/Ir(111) driven purely by molecule-molecule interactions is presented in Fig. S1. No influence of the graphene moiré is observable.

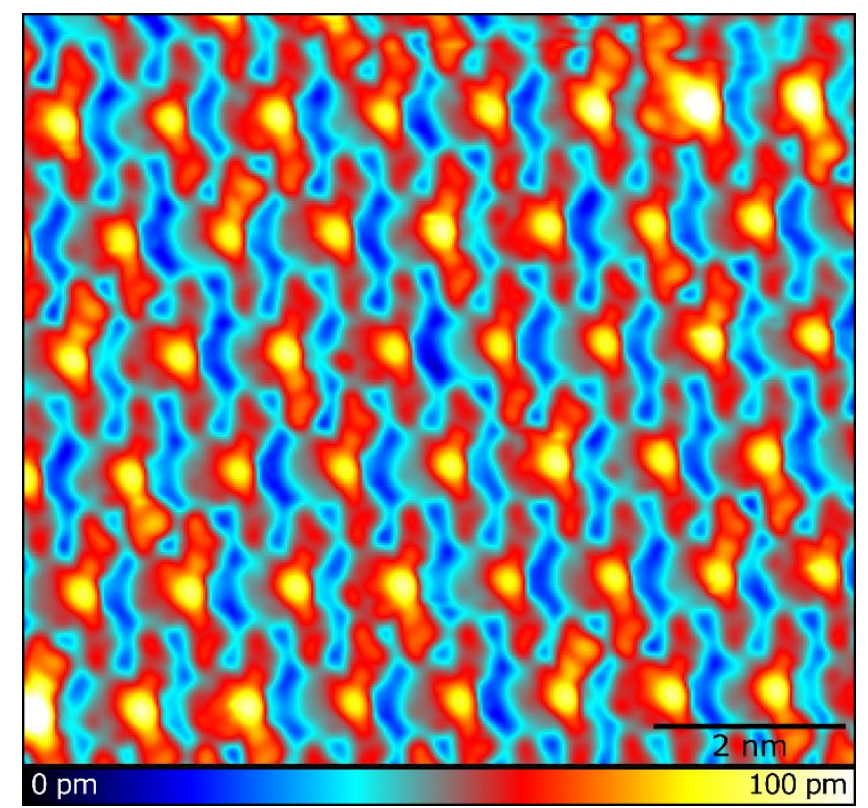

Figure S1: An STM image of a close-packed assembly of CoSal molecules adsorbed on pristine GR/Ir(111) obtained with a functionalized probe tip.

\section{Extraction of magnetization curves from field dependent $d I / d U$ maps}

This section explains the analysis of the data gathered in a variable external magnetic field $(\vec{B})$. The analyzed data set consisted of $d I / d U$ maps of an area presented in Fig. 1a at a bias voltage $U$ of $U=+450 \mathrm{mV}$ (where the molecules' Co centers are visible). The state of the Co center at this energy appears round in shape with a maximum in its middle. The maps had been obtained as a function of the externally 
applied magnetic field $(B=0.75 \mathrm{~T} \rightarrow 5.25 \mathrm{~T} \rightarrow-5.25 \mathrm{~T} \rightarrow 0.75 \mathrm{~T})$ with steps of $0.75 \mathrm{~T}$. The field sweep was accompanied by a drift in the XY-plane on the order of a few nanometers, therefore, the scanning area had to be adjusted accordingly. In order to compensate for the drift and small misposition errors we used an automated algorithm to find the maxima in the $d I / d U$ map of the molecules and extracted a spatially averaged $G$-value according to the following procedure:

1. User input of coarse coordinates for the CoSal molecule for each $d I / d U$ map (i.e. each $\vec{B}$-field).

2. An algorithm finds the maximum value of the $d I / d U$ signal within an area of $\sim 0.2 \mathrm{~nm}^{2}$ around these coordinates.

3. The $G$-value for the CoSal molecule is obtained by averaging the $d I / d U$ signal over an area of $\sim 0.1 \mathrm{~nm}^{2}$ around the maximum $d I / d U$ value.

4. The $G$-value for the $\mathrm{GR} / \mathrm{Fe}$ region is averaged over a molecule-free area of $\sim 0.7 \mathrm{~nm}^{2}$.

5. The error for each $G$-value is given by the standard deviation of the average.

\section{Movie}

The supplementary movie shows the complete data set as analyzed above. The first frames of the movie present the exact areas of interest from the STM topography image of Fig. 3a for the data extraction described above and used to create the graph in Fig. 3b. The upper right square contains the CoSal molecule area, while the lower left square contains the hcp area of the GR/Fe substrate. The corresponding boxes on the right present the recorded spin-resolved $d I / d U$ signal. In the following frames, we present the process of obtaining the magnetization curves. The spatial distribution of the spin-resolved $d I / d U$ signal changes as we change the externally applied $\vec{B}$-field. The $G$ value for the CoSal molecule is extracted from the square area marked with a white dashed line. The $G$ value for the $\mathrm{GR} / \mathrm{Fe}$ substrate is extracted from an area free of molecules.

Movie S1: Magnetization curve as presented in the graph of Fig. 2a along with the spatial distribution of the recorded spinresolved $d I / d U$ signal for each point.

\section{Strong interaction of CoSal with the GR/Fe substrate}

The parallel or antiparallel orientations of molecules' magnetic moment have been deduced from the behavior of the magnetometry curves recorded on other molecules at higher external magnetic fields of up to 6T. Parallelly oriented molecules have a lower conductance when the GR/Fe magnetic moment (and that of the molecule) is antiparallel to that of the SP-STM tip (Fig. S2a). After the GR/Fe switches, the molecule switches as well. It is the only change observed in the curve. The antiparallelly oriented molecules start with a higher 
conductance when the GR/Fe magnetic moment is antiparallel to that of the SP-STM tip (Fig. S2b). When the $\mathrm{GR} / \mathrm{Fe}$ switches at $5.25 \mathrm{~T}$ the molecule switches as well and the conductance drops. When we continue increasing the field by additional $0.75 \mathrm{~T}$ the conductance starts to rise again. It means that after the switch the magnetic moment of a molecule is being influenced by the external magnetic field and is being bent as in case of the molecule investigated in the main text. The molecules exhibiting either parallel (P) or antiparallel (A) oriented magnetic moments are marked in the topography image (Fig. S2c). The molecular assembly consists of 28 molecules in total. 16 molecules are magnetically parallel oriented with respect to the substrate and 5 are antiparallel oriented. For the 7 molecules which are not marked in the image, the signal-to-noise ratio in the magnetization curve data is not sufficient to make a clear assignment.
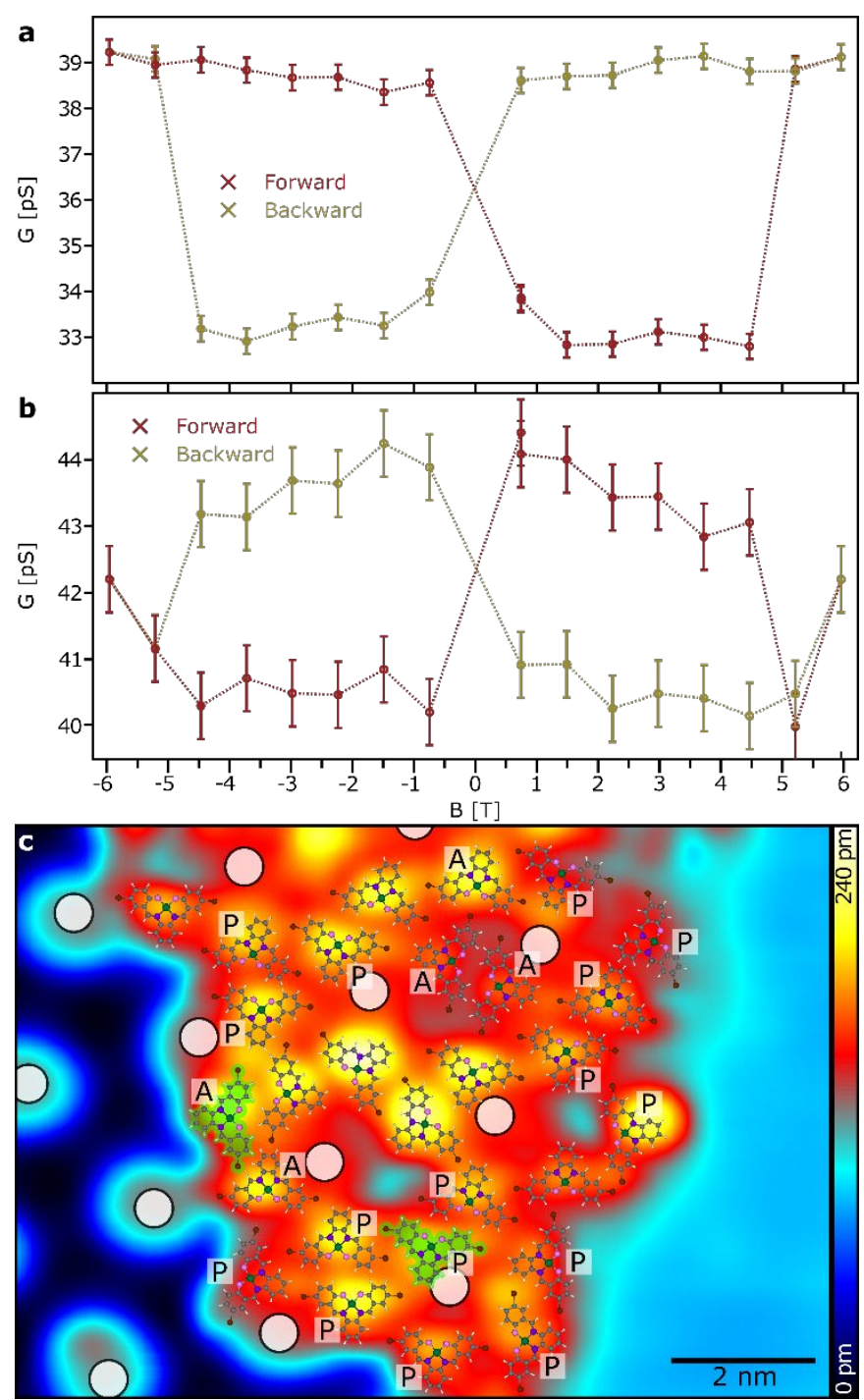

Figure S2: Spin-resolved differential tunneling conductance $G$ extracted from the measured $d I / d U$ signal averaged over the molecule's Co-metal center showing two different responses suggesting parallel (a) and antiparallel (b) alignment of the molecule's magnetic moment with respect to the GR/Fe substrate magnetization. The error bars correspond to the highest standard deviation of the spatially averaged $d I / d U$ signals. (c) An STM overview image of the molecular assembly adsorbed on $\mathrm{GR} / \mathrm{Fe}$ with overlaid models of molecules. Labels next to the molecules indicate parallel (P) or antiparallel (A) alignment of their magnetic moments with respect to the substrate magnetization direction. Molecules for which the magnetization curves presented in (a) and (b) were obtained are marked with green color. 


\section{Control experiment}

In order to validate the experiment, changes of the tip state must be excluded as it may be a source of a fake contrast. The procedure is based on the magnetic contrast being reversible, meaning the same differential conductance curve has to be registered when changing the $\vec{B}$-field back to its initial value. Fig. S3 presents such experimental data obtained on the molecule (Fig. S3a) and corresponding GR/Fe hcp area (Fig. S3b) as investigated in the main paper

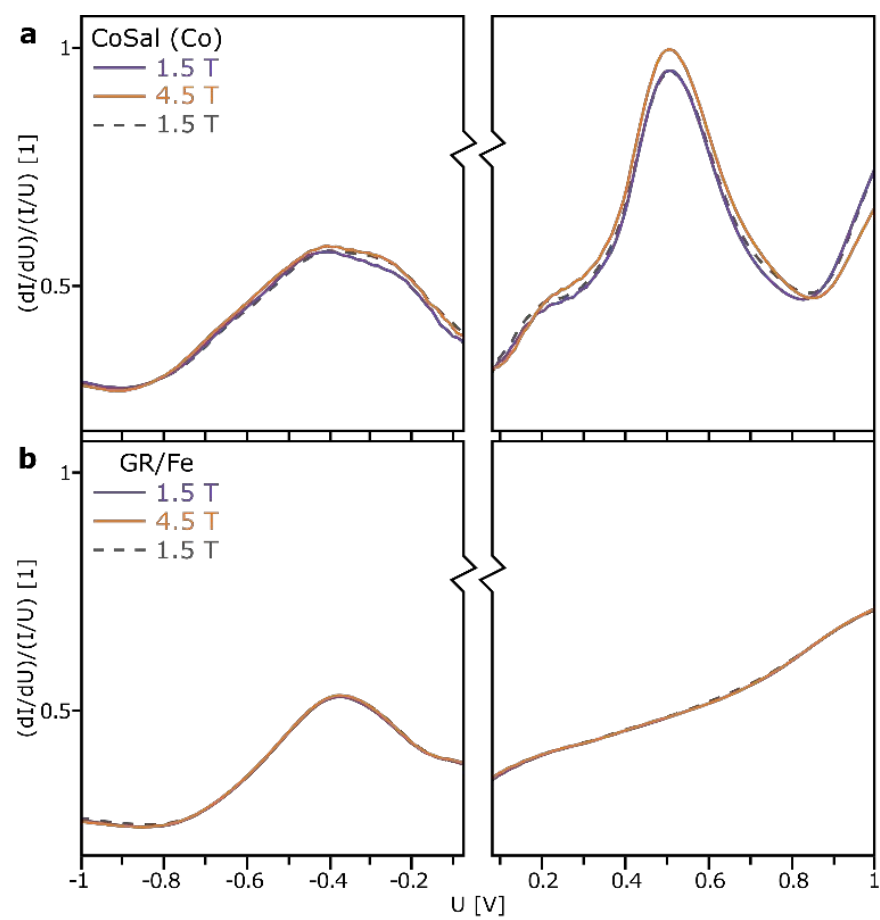

Figure S3: (a) Normalized point spectroscopy obtained on the Co metal center of a CoSal molecule in $B=1.5 \mathrm{~T}$, $B=4.5 \mathrm{~T}$ and again in $B=1.5 \mathrm{~T}$. (b) Corresponding normalized point spectroscopy obtained on a GR/Fe area next to the molecular assembly.

\section{Mixed spin polarization of CoSal molecule and GR/Fe after switching the GR/Fe magnetization direction.}

Here we present the influence of the GR/Fe on the measured SP-STS data on CoSal in the case of a remagnetization of the substrate. A sufficiently strong $\vec{B}$-field can align the magnetization direction of the GR/Fe substrate along its direction. Such a change significantly influences the substrate's LDOS and therefore the recorded point-spectroscopy data. In order to visualize this, we obtained the spectra at the same two places, i.e. one at the Co metal center of a CoSal molecule (Fig. S4a) and one at an hcp site of the GR/Fe substrate (Fig. $\mathrm{S} 4 \mathrm{~b}$ ), both in the same $\vec{B}$-field. A second set of spectra were obtained after magnetizing the sample with $B=6$ $\mathrm{T}$ and therefore, changing the magnetization direction of the GR/Fe substrate. We can observe that the differences are visible on both occupied and unoccupied states of the normalized spectra. The change of the 
GR/Fe magnetization direction and its LDOS influences the LDOS of the adsorbed CoSal molecule (see Fig. S4a). Therefore, in order to visualize the changes in the LDOS of a CoSal molecule responding to the applied $\vec{B}$-field it is important to prevent a change of the substrate's magnetization and perform the experiments within $B=[-4.5 \mathrm{~T}, 4.5 \mathrm{~T}]$ as described in the main part of the paper.

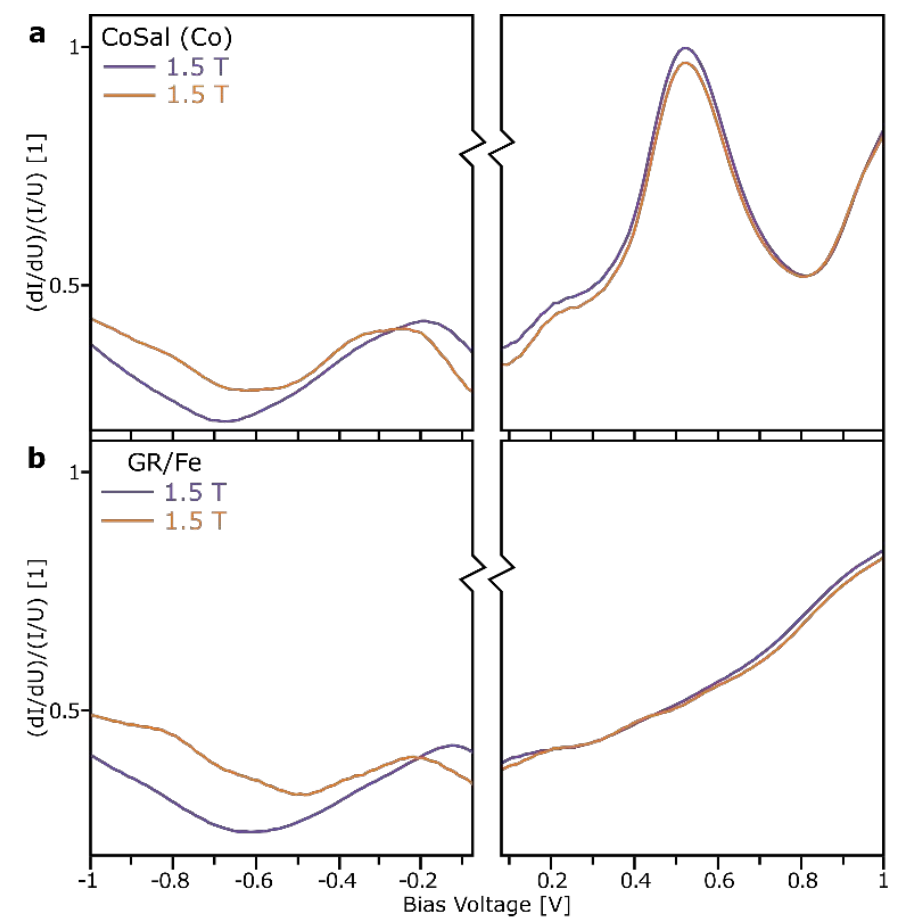

Figure S4: (a) Normalized point spectroscopy obtained on the Co metal center of a CoSal molecule in $B=1.5 \mathrm{~T}$ and again in the same field after magnetizing the sample in $B=6 \mathrm{~T}$. (b) Corresponding normalized point spectroscopy obtained on a $\mathrm{GR} / \mathrm{Fe}$ area next to the molecular assembly.

\section{Source of the spin-polarized signal}

In case of tunneling to weakly hybridized molecules adsorbed on conducting surfaces, there are two mechanisms to consider. First, a resonant tunneling involving an electron tunneling in two stages: from the probe tip to the molecule and from the molecule to the substrate. Second, a non-resonant tunneling from the tip directly to the substrate over the area occupied by the molecule in which the tunneling barrier is changed due to the presence of the molecule. It effectively modifies the dielectric constant and lowers the tunneling barrier.

In the case of the experiment described in this manuscript, it is in fact a combination of both above mentioned mechaniss. The resonant tunneling over the LUMO+1 state is evident in tunneling spectroscopy data (see Fig. 3a). The state is short lived as it is very broad in the tunneling spectrum. The tunneling from the state to the substrate is very efficient, and therefore we do not observe any charging effects. Tunneling also occurs between the tip and the substrate as evident by the non-zero conductance in the HOMO-LUMO gap. This enables the high-resolution imaging of the molecule's back-bone structure as can be observed in Fig. 1b. 
In the spin-polarized tunneling experiment as presented here, the tunnel junction is composed of two magnetic electrodes with a magnetic quantum dot in-between. In terms of tunneling, the quantum dot (CoSal molecule) is strongly coupled to one of the electrodes, namely the GR/Fe substrate. Because of the energy mismatch of the spin-polarized states in $\mathrm{GR} / \mathrm{Fe}$ and in the CoSal molecule the overall spin-asymmetry in conductance is rather low. 